\title{
TWO PSEUDOBULGES IN THE “BOXY BULGE” GALAXY NGC 5746
}

\author{
John C. Barentine and JoHn Kormendy \\ Department of Astronomy, University of Texas at Austin, 1 University Station C1400, Austin, TX 78712-0259, USA \\ Received 2012 May 4; accepted 2012 May 30; published 2012 July 18
}

\begin{abstract}
Galaxy formation and growth under the $\Lambda \mathrm{CDM}$ paradigm is expected to proceed in a hierarchical, bottom-up fashion by which small galaxies grow into large galaxies; this mechanism leaves behind large "classical bulges" kinematically distinct from "pseudobulges" grown by internal, secular processes. We use archival data (Spitzer Space Telescope $3.6 \mu \mathrm{m}$ wavelength, Hubble Space Telescope $H$-band, Two Micron All Sky Survey $K_{s}$-band, and Sloan Digital Sky Survey gri-band) to measure composite minor- and major-axis surface brightness profiles of the almost-edge-on spiral galaxy NGC 5746. These light profiles span a large range of radii and surface brightnesses to reveal an inner, high surface brightness stellar component that is distinct from the well-known boxy bulge. It is well fitted by Sérsic functions with indices $n=0.99 \pm 0.08$ and $1.17 \pm 0.24$ along the minor and major axes, respectively. Since $n<2$, we conclude that this innermost component is a secularly evolved pseudobulge that is distinct from the boxy pseudobulge. This inner pseudobulge makes up $0.136 \pm 0.019$ of the total light of the galaxy. It is therefore considerably less luminous than the boxy structure, which is now understood to be a bar seen nearly end-on. The infrared imagery shows further evidence for secular evolution in the form of a bright inner ring of inner radius $9.1 \mathrm{kpc}$ and width $1.6 \mathrm{kpc}$. NGC 5746 is therefore a giant, pure-disk SB(r)bc galaxy with no sign of a merger-built bulge. We do not understand how such galaxies form in a $\Lambda$ CDM universe.
\end{abstract}

Key words: galaxies: bulges - galaxies: evolution - galaxies: individual (NGC 5746)

\section{INTRODUCTION}

The $\Lambda$ CDM paradigm is based on the observation of dark matter concentrations ("halos") into which baryons fall, cool, and form stars. Galaxies are built by the hierarchical merging of smaller halos in a bottom-up fashion (White \& Rees 1978). This implies frequent "major" mergers in the early universe between objects of comparable mass. Mergers are understood to scramble disks into elliptical galaxies (Toomre 1977; Schweizer 1990) and are accompanied by cold-gas dissipation and starbursts in the smaller galaxies but not in the biggest galaxies that can hold onto X-ray gas (see Kormendy \& Kennicutt 2004 for a review). The rate of major mergers peaked at $z \sim 1.2$ (Ryan et al. 2008), and since that time the universe has been in transition from merger-dominated galaxy evolution to slow ("secular") internal evolution (Kormendy \& Kennicutt 2004).

In spite of the large amount of merger violence at early times, almost two-thirds of the most massive galaxies in the nearby universe (field environments like the Local Group) show no classical bulge at all (Kormendy et al. 2010; Fisher \& Drory 2011; see Peebles \& Nusser 2010 for a review). Thus, most field galaxies show no sign that they experienced a major merger at least since the time of the merger rate peak. It is difficult to understand how stellar disks that were already in place at $z \sim 1$ survived until today without being converted into- or at least augmented by - a classical bulge (Kormendy et al. 2010). Here, it is important to note that we do not have the freedom to postulate bulges whose properties make them easy to hide. Bulges satisfy well-defined "fundamental plane" parameter correlations (see Kormendy \& Bender 2012 for the most recent version). Low-luminosity classical bulges are small, but they have high surface brightnesses and are described by Sérsic functions (Sérsic 1968) with indices $n \simeq 2.5$ that make them hard to hide and easy to identify. Thus, we can be confident that, while $\Lambda \mathrm{CDM}$ performs well on large scales, the large fraction of nearby, giant galaxies that show little evidence of major mergers presents a challenge to our understanding of galaxy evolution on $10 \mathrm{kpc}$ scales (e.g., Abadi et al. 2003; Governato et al. 2010; Kormendy et al. 2010; Peebles \& Nusser 2010).

The magnitude of this challenge is underestimated if effectively bulgeless galaxies are undercounted because pseudobulges are mistaken for classical bulges. Prior to the development of the secular evolution picture (especially Kormendy 1993; Kormendy \& Kennicutt 2004), this routinely happened even for face-on galaxies with dynamically disky pseudobulges (e.g., NGC 4736; see the above references). But identification of classical bulges in edge-on galaxies is particularly tricky. "Box-shaped bulges" such as that in NGC 4565 (Kormendy $\&$ Barentine 2010) are made of old stars and clearly bulge out above and below the edge-on disk plane. It is understandable that, in early papers, they were thought to be classical bulges which happen to have a box-shaped peculiarity in their structure. But now we know that box-shaped bulges are galaxy bars that are seen edge-on (Combes \& Sanders 1981; Combes et al. 1990; Pfenniger \& Norman 1990; Pfenniger \& Friedli 1991; Raha et al. 1991; Athanassoula \& Misiriotis 2002; Athanassoula 2005; Shen et al. 2010). Bars form by gravitational instabilities in galaxy disks. The above papers show that, once a bar is well formed, it buckles vertically and rapidly turns into a thick structure that looks like a box-shaped bulge when seen edge-on. Heating of stars whose vertical motions are in resonance with the bar density wave further contributes to the thick, boxy-distorted structure. Also, a splitting of gas rotation velocities in edge-on boxy bulges (a "figure 8" shape of spectral emission lines) is a robust signature of gas flow in an edge-on bar and further cements our view of these boxy structures as bars (Kuijken \& Merrifield 1995; Merrifield 1996; Merrifield \& Kuijken 1999; Bureau \& Freeman 1999). Thus, a "boxy bulge" in an edge-on object would be identified as the galaxy's bar if it were seen more face-on. Since it is really a part of the disk, we call such structures "boxy pseudobulges." This means that the 
true classical-bulge-to-total luminosity ratios of galaxies such as NGC 4565 are much smaller than the values $\sim 1 / 3$ that are measured (e.g., Simien \& de Vaucouleurs 1986) when the boxy structure is identified as the bulge.

We demonstrated this effect for the nearly edge-on spiral NGC 4565 in Kormendy \& Barentine (2010). Its center has a boxy photometric signature previously identified as a classical bulge (Simien \& de Vaucouleurs 1986). But we found that this component is well fitted by a Sérsic function with index $n=1$. This demonstrates that it does not have the characteristics of a merger-built classical bulge (Kormendy \& Kennicutt 2004; Fisher \& Drory 2008). It is consistent with a bar seen nearly end-on. Mid-infrared observations penetrate the thick midplane dust, revealing the true central component in this galaxy. It is a pseudobulge whose scale height is smaller than that of the outer disk. Its small pseudobulge-to-total $(P B / T)$ ratio, $0.061_{-0.008}^{+0.009}$, means that the disk and its secularly built structures completely dominate this galaxy. NGC 4565 is a massive galaxy; its rotational speed is $\sim 255 \mathrm{~km} \mathrm{~s}^{-1}$ interior to its outer warp (Rupen 1991). This result is especially hard to understand because it is easier to make bulgeless galaxies via feedback mechanisms (Governato et al. 2010) when the resulting galaxy is a dwarf. Thus, NGC 4565 and galaxies like it are problematic in the context of hierarchical assembly models.

We know that galaxies like NGC 4565 are not rare (Kormendy et al. 2010; Fisher \& Drory 2011). Previous efforts to measure $B / T$ ratios of galaxies by way of light profile decompositions may have resulted in overestimates of the bulge contribution in galaxies at moderate to high inclinations. This could mask the true number of essentially bulgeless galaxies in the universe. The problem of apparently bulgeless galaxies becomes more acute because most decomposition work is now done in an automated fashion and in two dimensions. It is difficult for these analyses to cope with patchy internal obscuration. Here, we use carefully constructed one-dimensional (1D) light profiles to address this problem.

The aim of this paper is to determine for NGC 5746, i.e., an additional normal, edge-on disk galaxy with a boxy bulge, the fraction of the total galaxy luminosity that is contributed by the boxy structure and by any additional, disky pseudobulge near the center. This requires making a clear photometric distinction between structural components in circumstances compromised by strong dust absorption. To this end, we determine the $P B / T$ and the pseudobulge scale height. In Section 2, we describe the selection of this galaxy and outline the method by which we constructed minor- and major-axis light profiles from photometric data spanning a range of wavelengths from the optical to the mid-infrared. We present the profiles in Section 3 and compare them with previous studies. The galaxy's $P B / T$ ratio is calculated using these profiles, after subtracting fits to the other structures seen in the profiles. We show that NGC 5746 is a massive disk galaxy in which the bulge does not dominate the light profile at any radius. We summarize our results in Section 4.

\section{METHOD}

\subsection{Target Selection}

We searched various lists of edge-on galaxies and applied the following selection rules: candidate objects must (1) be nearby (distance $D \lesssim 75 \mathrm{Mpc}$ ), (2) be highly inclined (inclination $i \gtrsim$ $\left.85^{\circ}\right)$, (3) show minimal, if any, indication of recent interaction with other galaxies, (4) have available data over a wide range of wavelengths and resolutions, and (5) be relatively free of dust.
NGC $5746\left(\alpha_{2000.0}=14^{\mathrm{h}} 44^{\mathrm{m}} 56.005, \delta_{2000.0}=+01^{\circ} 57^{\prime} 17^{\prime \prime} .06\right)$ meets essentially all of these requirements. It is classified as an $\mathrm{SAB}(\mathrm{rs}) \mathrm{b}$ ? in the Third Reference Catalog of Bright Galaxies (RC3; de Vaucouleurs et al. 1991). Measurements reported in the literature give a mean distance of $27.6 \mathrm{Mpc}$ with a $1 \sigma$ dispersion of 2.5 Mpc (Tully 1988; Willick et al. 1997; Rand \& Benjamin 2008; Tully et al. 2008; Springob et al. 2009; Tully et al. 2009). The galaxy is inclined to the line of sight by 83.9 and has a rather large maximum circular velocity of $318.5 \pm 9.8 \mathrm{~km} \mathrm{~s}^{-1}$. The inclination and velocity were obtained from HyperLEDA ${ }^{1}$ and the circular velocity is corrected for inclination. While not as highly inclined as we would prefer, the observed angle has the benefit of reducing the effect of the dust in the central region. This galaxy also has the largest amount of archival photometric data of the candidate objects we considered.

NGC 5746 has been shown previously to contain kinematic evidence for the presence of a bar (Kuijken \& Merrifield 1995; Bureau \& Freeman 1999), manifesting itself in the apparent box-like shape of the "bulge" in optical images. We confirm the existence of the bar in observations reported here. The question remains: If the apparent bulge is actually the photometric signature of the bar, then where is the bulge in this galaxy?

\subsection{Data and Calibrations}

In many edge-on disk galaxies other than S0 galaxies, extinction at optical wavelengths is very large along sightlines through the disk midplane and could hide structures with scale heights smaller than that of the dust. Observations in the near- and mid-infrared can help overcome this problem. To see through the dust, we used $3.6 \mu \mathrm{m}$ images made with the Infrared Array Camera (IRAC; Fazio et al. 2004) on board the Spitzer Space Telescope to measure the minor- and major-axis light profiles NGC 5746. The spatial resolution of IRAC is insufficient to extend the light profiles to the smallest radii, so in the innermost region we augmented the profile with $H$-band data from the Hubble Space Telescope Near-Infrared Camera Multi-Object Spectrometer (HST/NICMOS; Thompson 1994). At large radii, where dust is less of a problem and maximizing the signal-to-noise ratio $(\mathrm{S} / \mathrm{N})$ becomes more important, we used data from two large sky surveys: the Two Micron All Sky Survey (2MASS; Skrutskie et al. 2006) and the Sloan Digital Sky Survey (SDSS; York et al. 2000). Filters, pixel scales, and fields of view of each telescope and instrument are given in Table 1 . We briefly summarize the calibration procedures for each data source.

The IRAC data were reduced by the Spitzer Science Center (SSC) using software pipeline version S14.0.0. The reduction steps include subtracting the bias level and dark current, flat fielding, and performing sky subtraction. We used the final, mosaicked versions of the images containing all pointings of the telescope at a given location and time of observation.

NICMOS data were calibrated using version 4.4.0 of the CALNIC reduction pipeline (Bushouse 1997). The code applies basic corrections to the data, including dark current subtraction, corrections for detector nonlinearity, and flat fielding. After all images in an "association" of data are processed in this manner, a second stage creates mosaics of overlapping images and subtracts a scalar background ("sky") value. However, proper sky subtraction of images of extended sources is severely impacted by the instrument's small field of view. NGC 5746 overfills the NICMOS frame such that at no location is the

\footnotetext{
The Lyon-Meudon Extragalactic Database: http://leda.univ-lyon1.fr/.
} 
Table 1

Properties of Data Sources

\begin{tabular}{lcccc}
\hline \hline Telescope & Instrument & Field of View & Scale & Filters \\
& & $\left({ }^{\prime}\right)$ & $1.2 \times 1.2$ & Channel $1(3.6 \mu \mathrm{m})$ \\
Spitzer $0.85 \mathrm{~m}$ & IRAC & $5.22 \times 5.22$ & F160W $(1.6 \mu \mathrm{m})$ \\
HST $2.4 \mathrm{~m}$ & NICMOS & $0.3 \times 0.3$ & $0.076 \times 0.075$ & $K_{s}$ \\
2MASS $1.3 \mathrm{~m}$ & 2MASS Camera & $8.5 \times 8.5$ & $2.0 \times 2.0$ & $g, r, i$ \\
SDSS $2.5 \mathrm{~m}$ & Imager & $13.51 \times 8.98^{\mathrm{a}}$ & $0.4 \times 0.4$ & $\mathrm{~g}$ \\
\hline
\end{tabular}

Note. ${ }^{a}$ Dimensions are given for a single image frame.

true sky level reached; the automated data reduction procedures typically overcorrect for sky by subtracting a value higher than the true sky level, resulting in negative pixel values in the corners of frames. We accounted for this by locating NICMOS frames from other programs taken as close in time as possible to our galaxy observations, typically within one day, and measuring real sky values from frames that did not contain large, extended objects. The "sky" value recorded in the headers of our galaxy images removed by CALNIC is added back to the pixels in our images and the measured sky value subtracted off.

The 2MASS data were calibrated nightly during survey operations by observing standard star fields at regular intervals. Photometry of the standard stars was used to derive the extinction coefficients and photometric zero points in each of the three survey passbands as a function of time throughout a given night. The survey observations did not permit absolute calibration of the 2MASS photometric system. However, Cohen et al. (2003) offer a calibration tied to Vega on the "Cohen-Walker-Witteborn" system from computation of relative spectral response curves. To place our fluxes on the 2MASS system, we used the zero points computed by the reduction pipeline and written into the image headers.

Photometric data from SDSS were calibrated using the PHOTO pipeline (Lupton et al. 2001), which gathers astrometric data, obtains the extinction and photometric zero point on the night the data were collected, renders the drift-scan images into a series of postage stamps, and estimates the flat field vectors, bias drift, and the sky level for each. Corrected frames are produced using this information. We did not make use of SDSS-generated PSF or Petrosian-fitting photometry, but rather performed our own surface photometry on corrected frames.

\subsection{Surface Photometry}

Images from a given source were first prepared by correcting for any background gradients and cleaning contaminating pixels from sample regions. Systematic variations in the background were removed with the IRAF ${ }^{2}$ task mscskysub, which fits a polynomial or spline of arbitrary order to a two-dimensional surface. We specifically used second-order polynomials. Contaminants consisting of cosmic rays, foreground stars, and background objects were removed by computing the median of pixels in a user-defined region around the source; pixels varying from the median by more than $1.5 \sigma$ were replaced by the median. This cleaning was done carefully by hand, and individual, problematic pixels were replaced on a case-by-case basis. Extended objects were treated by interpolating over pixels in a user-defined box around an object with a second-order polynomial and replacing the pixel values in the box with those of the

\footnotetext{
2 IRAF is distributed by the National Optical Astronomy Observatory, which is operated by the Association of Universities for Research in Astronomy
} (AURA) under cooperative agreement with the National Science Foundation.

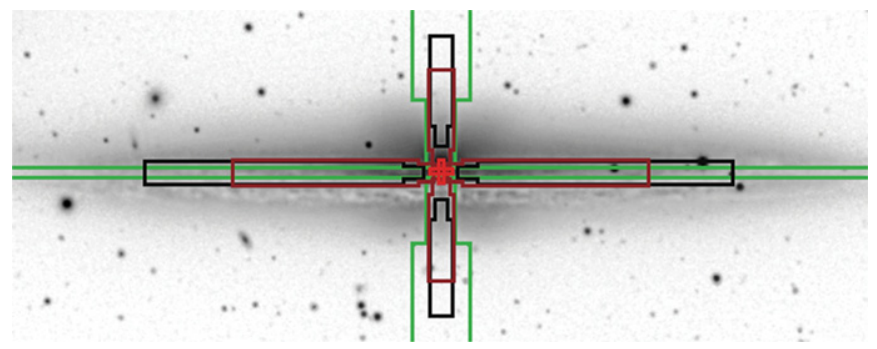

Figure 1. $8100 \mathrm{~s} R$-band image of NGC 5746, rendered with an inverted color map, onto which has been superimposed a set of colored overlays representing the effective sizes and shapes of the cut boxes used in performing surface photometry. The image has been rotated such that the major axis is aligned with the image rows. The colors of the boxes indicate the data sources, SDSS (green), $H S T$ /NICMOS F160W (red), 2MASS $K_{s}$ (brown), and Spitzer/IRAC $3.6 \mu \mathrm{m}$ (black), and match the colors of the data points in the minor- and major-axis light profiles presented in Figures 3 and 4 . The radial extent of the boxes reflects the radial range of points plotted in the light profiles.

fit. This approach works well for moderately bright stars but breaks down for the brightest objects; the affected pixels were edited out of the extracted 1D light profiles by hand.

We performed surface photometry on NGC 5746 by taking rectangular cuts along its minor and major axes, supplemented where possible with ellipse fits of isophotes that served as a check on the cuts. We generally avoided ellipse fitting in favor of cuts because the isophotes are far from elliptical over a large range of radii, and ellipse fits tend to fail in the presence of significant midplane dust absorption. For each data source, we investigated a range of cut widths over different radius ranges in order to construct light profiles of the highest possible $\mathrm{S} / \mathrm{N}$ at large radii while preserving resolution at small radii. Consequently, the cut boxes effectively had stairstep shapes tapering to progressively narrower widths at smaller radii; the effective dimensions and orientations of these cut boxes are illustrated in Figure 1, in which they are superimposed over an optical image of the galaxy. The sky level in each image was determined by sampling regions at large radii as free from contamination by foreground and background objects as possible. 1D profiles were extracted by block-averaging pixels along the short dimension of a cut box in IRAF. In order to mitigate the effect of midplane dust absorption where it interferes with the minor-axis light profile, we excised points by hand from the profile that were obviously impacted adversely by the dust. For the major-axis profile, we defined the cut box parameters to carefully avoid the dust lane (see Figure 1 for placement).

All photometric data were given an absolute calibration by tying them to the 2MASS $K_{s}$ points in order to place them on a common photometric system. To assemble a composite light profile from a number of data sources, arbitrary constants were added to light profiles generated for each source individually to bring data points into coincidence with those of the 2MASS 


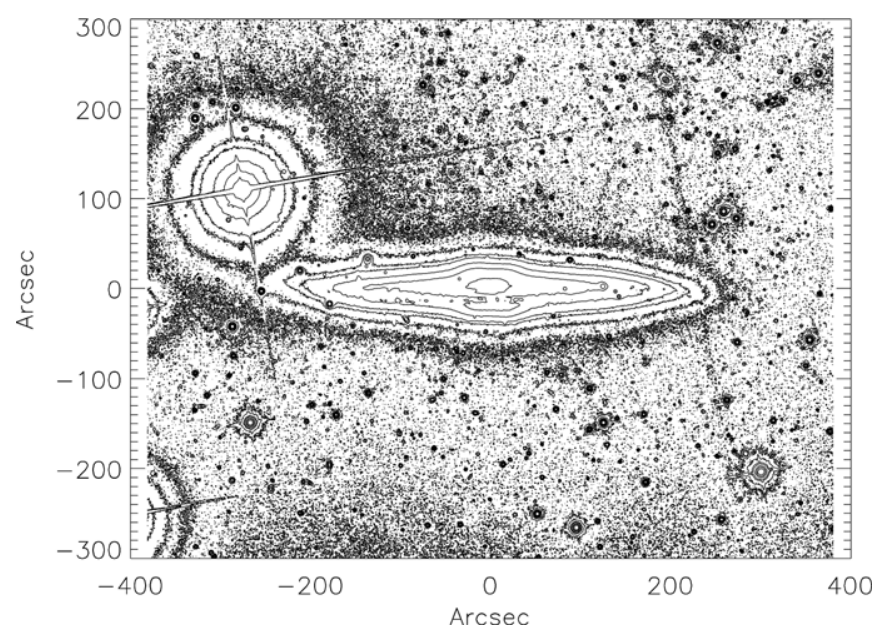

Figure 2. Brightness contour plot of NGC 5746 made from the $8100 \mathrm{~s} R$-band image of NGC 5746 in Figure 1. The contours are at $-9,10,30,75,125,325$, 800 , and 4000 ADU above the mean sky background as measured $\sim 10^{\prime}$ from the center of the galaxy along its minor axis. The isophotes nearest the sky level do not show any obvious indication of an extended halo. The bright star at upper left is HD 129827.

profile over the radius regime in which they overlap. Profiles tend to plateau at small radii for data sources with poor spatial resolution; these points were trimmed from the final profile of each object. Additionally, points were trimmed at large radii at which the instrumental sensitivity rapidly diminishes, otherwise leading to an underestimate of the surface brightness. We present the final versions of the light profiles for each object with symbol colors corresponding relatively to the wavelengths of the data sources to help guide the eye.

\subsection{ID Radial Profile Decomposition}

After extracting the profiles, we decomposed them into three components: a Sérsic function for the central pseudobulge, another Sérsic function for the boxy bulge, and an outer exponential representing the disk. The choice of an exponential is consistent with the van der Kruit \& Searle (1981) model of the disk as a locally isothermal sheet. All components were fitted with a $\chi^{2}$-minimization algorithm employing the simplex optimization method. Our fitting code allows for the simultaneous decomposition of a given profile into a single Sérsic and single exponential function with five free parameters: the radius and surface brightness of the bulge $\left(R_{n}, \mu_{n}\right)$, the radius and surface brightness of the disk $\left(R_{d}, \mu_{d}\right)$, and the Sérsic index, $n$.

We considered the possibility that our observations might reach a limiting magnitude sufficient to reveal the presence of an extended halo around NGC 5746, thereby informing the choice of the functional form of the fit to the largest radii in our profiles. We searched for evidence of an extended halo in a deep $(8100 \mathrm{~s}) R$-band image of NGC 5746 obtained with the WIYN $0.9 \mathrm{~m}$ telescope and S2KB CCD camera at Kitt Peak National Observatory over two observing seasons in 2011-12. Brightness contours of the image are shown in Figure 2. Despite contamination of the faintest isophotes from the bright nearby star HD 129827, we do not find any convincing detection of a halo to a limiting surface brightness of $\sim 23 \mathrm{mag} \mathrm{arcsec}^{-2}$ in $K_{s}$. Thus, we do not include an explicit halo component in the light profile fits at large radii. Specific details of the fit, including its functional form, are not critical to the analysis presented here. We also did not include a component representing a central

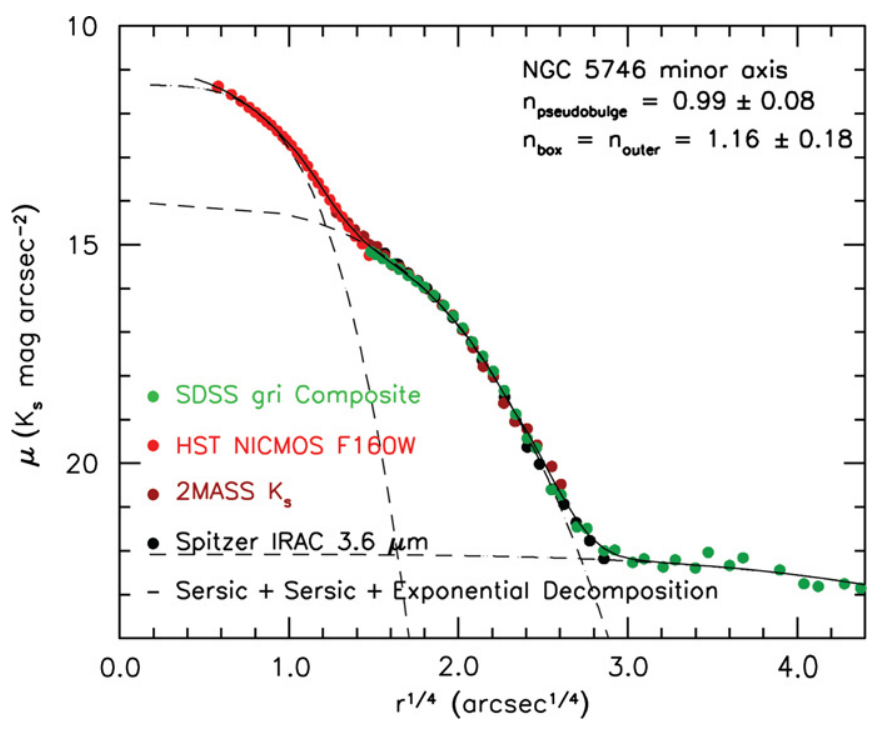

Figure 3. Minor-axis light profile of NGC 5746 from the following data sources: combined SDSS gri (green points), HST/NICMOS F160W (red points), 2MASS $K_{s}$ (brown points), and Spitzer/IRAC $3.6 \mu \mathrm{m}$ (black points). A Sérsic-Sérsic-exponential decomposition is overplotted as dashed lines, representing the inner pseudobulge, boxy bar, and outer halo, respectively. The solid line represents the sum of these three components.

Seyfert nucleus or a nuclear star cluster in the fit at the smallest radii. A bright central point was seen in our NGC 4565 data but we did not attempt to fit it, whereas we do not see a corresponding point in the NGC 5746 images.

We subtracted the disk exponential and box Sérsic fits from a given profile, leaving the profile of the pseudobulge itself. Once the pseudobulge light profiles were obtained for both axes, the total luminosity of the pseudobulge was obtained by integrating the light in each direction and adding the results. A proper comparison of light profiles of the minor and major axes of an edge-on galaxy with a boxy pseudobulge should take into account the fact that the box typically has an axial ratio other than 1 . As a result, a feature seen at radius $r$ along the minor axis will be seen at radius $(b / a) r$ along the major axis, where $a$ and $b$ are the sides of the box. Our major-axis light profiles are shifted in radius by a factor of 1.4 , the axial ratio of the box measured from the SDSS gri composite image, placing the profiles on a common spatial scale.

\section{RESULTS}

The minor- and major-axis light profiles we obtain for NGC 5746 are shown in Figures 3 and 4, respectively. A simultaneous decomposition of the box and exponential disk yielded Sérsic indices of $n=1.16 \pm 0.18$ along the minor axis and $1.78 \pm 0.25$ along the major axis. Fitting the box along the major axis is complicated by the presence of a bright ring, described below, seen in the infrared imagery. Interior to this ring, at radii $1.8 \operatorname{arcsec}^{1 / 4} \lesssim r^{1 / 4} \lesssim 2.7 \operatorname{arcsec}^{1 / 4}$, the infrared flux is low relative to the optical flux.

The central pseudobulge was fitted with a single Sérsic component over only the inner $\sim 2^{\prime \prime}$, corresponding roughly to the angular extent of the NICMOS data. The Sérsic indices of the central pseudobulge are $0.99 \pm 0.08$ along the minor axis and $n=1.17 \pm 0.24$ along the major axis. Prior evidence of a bar in NGC 5746 (Bureau \& Freeman 1999) is bolstered by our finding of $n<2$ for the box. Therefore, the true bulge in NGC 5746 is a pseudobulge, consistent with both our results for 


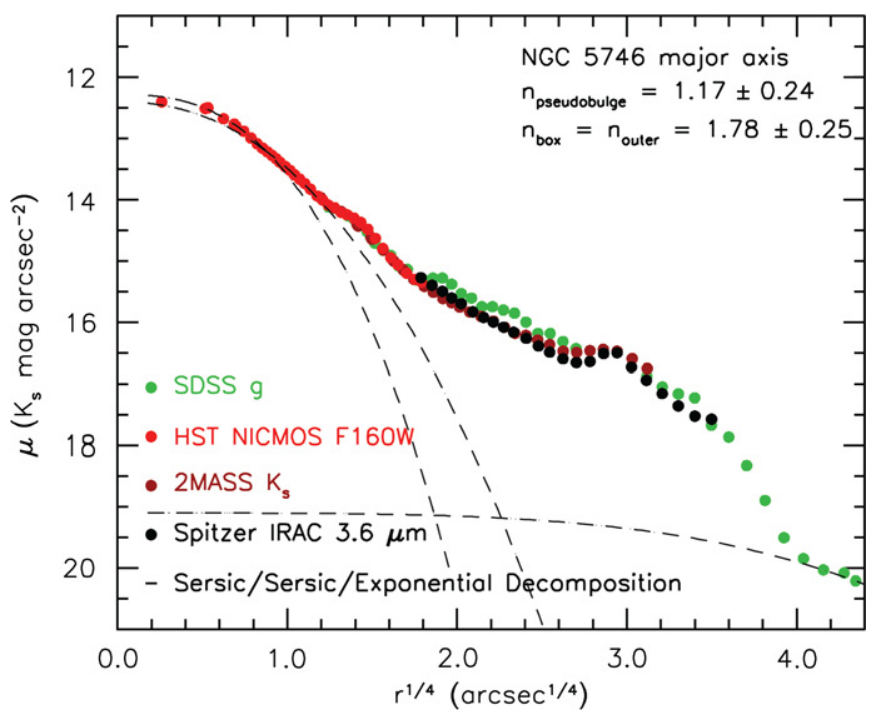

Figure 4. Major-axis light profile of NGC 5746. The data sources are the same as in Figure 3. A Sérsic-Sérsic-exponential decomposition is overplotted as dashed lines.

NGC 4565 and the observational definition in Fisher \& Drory (2008) that bulges with $n \lesssim 2$ are pseudobulges, not classical bulges. It is also evident that, as in NGC 4565, the apparent "boxy bulge" of NGC 5746 is in fact a bar seen nearly end-on. The emerging picture of NGC 5746 is that viewed face on, it would have a bar and an inner ring much like those inferred in NGC 4565.

The fits give additional information about the nature of the central pseudobulge. The best Sérsic fits yield a scale height of $0.74 \pm 0.10$ along the minor axis and a scale length of $0^{\prime \prime} .64 \pm$ 0 '.20 along the major axis. At our adopted distance to NGC 5746, these correspond to $100 \pm 13 \mathrm{pc}$ and $86 \pm 27 \mathrm{pc}$, respectively. From the fit to the boxy bar plus the disk, we compute a scale height of $755 \pm 145 \mathrm{pc}$. We measured the thick disk scale height as a function of radial distance along the major axis from the IRAC $3.6 \mu \mathrm{m}$ data; the cuts we used are shown superimposed on the IRAC image in Figure 5. Again, the cut box widths vary to preserve resolution at small radii along the major axis and $\mathrm{S} / \mathrm{N}$ at larger radii. The mean of eight measurements of the thick disk scale height at $r>38^{\prime \prime}(51 \mathrm{kpc})$ is $8^{\prime \prime} .6 \pm 0$. $7(1.2 \pm 0.1 \mathrm{kpc})$. For comparison, we found for NGC 4565 a pseudobulge scale height of $90 \mathrm{pc}$, a boxy bar plus disk scale height of $740 \mathrm{pc}$, and a thick disk scale height of $1.03 \mathrm{kpc}$.

We integrate the fits to the various components of the light profiles and find a mean value and $1 \sigma$ scatter of $0.136 \pm$ 0.019 for the $P B / T$ of NGC 5746. In Table 2, we show the Sérsic $n$ values measured along both minor and major axes and the resulting $P B / T$ ratio for this galaxy along with those reported for NGC 4565 in Kormendy \& Barentine (2010) for purposes of comparison. Measurements of the index of the boxy component of the apparent bulge and the true (pseudo)bulge are given along with the consequent $P B / T$ ratios. The pseudobulge in NGC 5746 contributes relatively little light to the galaxy. Balcells et al. (2007), following a similar procedure, quote a Sérsic index of $n=1.55 \pm 0.14$ and a $B / D$ ratio of 0.1 for this galaxy. While we recognize the care with which their measurements were made, we note that in neither case was allowance made for a nuclear component which could introduce uncertainty into values of the Sérsic $n$. However, the differences are not enough to affect our fundamental conclusion, one which

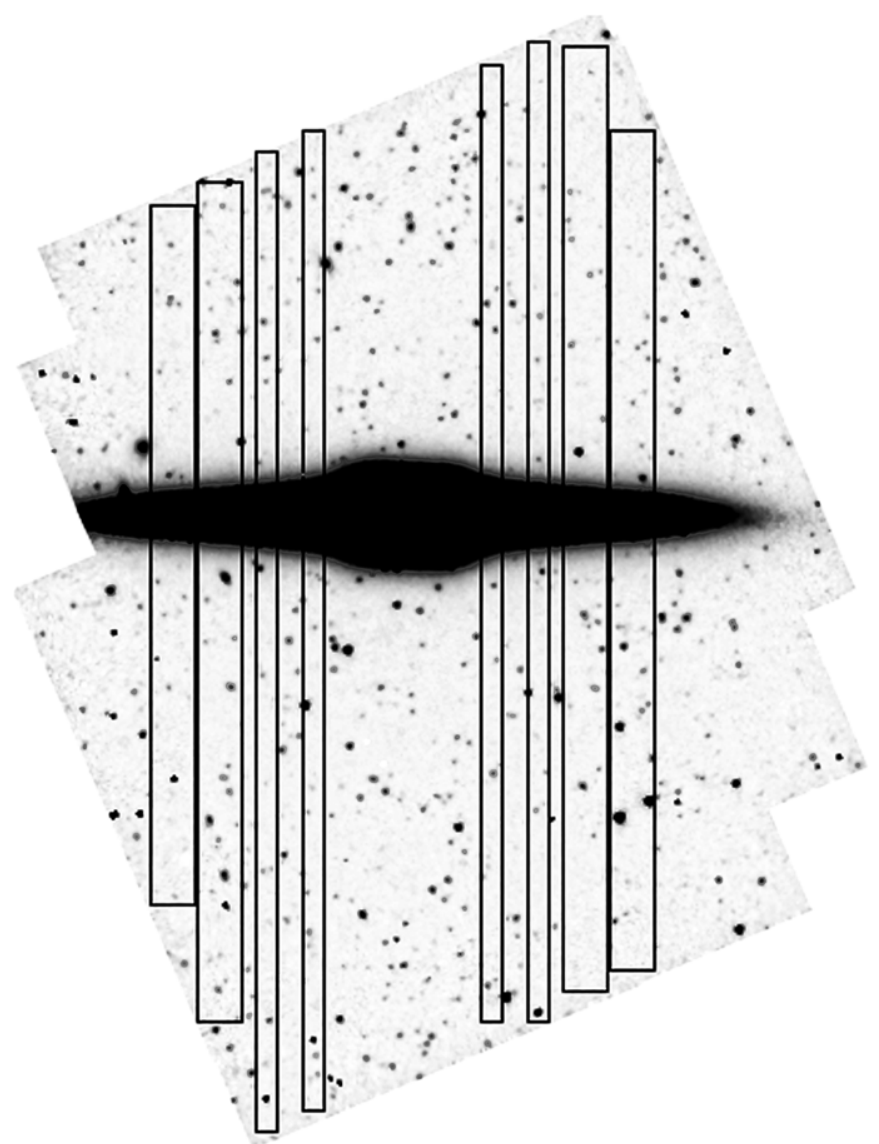

Figure 5. IRAC $3.6 \mu \mathrm{m}$ image of NGC 5746, rendered with an inverted color map, showing the dimensions and extent of the cut boxes used to extract minor axis light profiles for determination of the thick disk scale height as a function of radial distance along the major axis. The image has been rotated such that the major axis is aligned with the image rows.

the measurements of Balcells et al. support-the bulge in NGC 5746 is a pseudobulge.

Figure 6 shows the $3.6 \mu \mathrm{m}$ and $8 \mu \mathrm{m}$ IRAC images along with the SDSS gri composite for optical context, each individually stretched to emphasize the inner ring. Inspection of the $3.6 \mu \mathrm{m}$ image clearly indicates a bright ring with an inner radius of $\sim 57^{\prime \prime}(9.1 \mathrm{kpc})$ and an average radial width of $10^{\prime \prime}(1.6 \mathrm{kpc})$ as measured at the tangent points. The ring is clearly present in Figure 4 as a shallow rise in the major-axis light profile between $2.7 \operatorname{arcsec}^{1 / 4} \leqslant r^{1 / 4} \leqslant 3.2 \operatorname{arcsec}^{1 / 4}$ and is slightly brighter at its outer edge than inner edge, particularly at optical wavelengths. In the IRAC $8 \mu \mathrm{m}$ band, associated with emission by polycyclic aromatic hydrocarbons (PAHs; Léger \& Puget 1984; Allamandola et al. 1985, 1989; Puget \& Léger 1989; Tielens et al. 1999, 2000), the ring is considerably brighter, indicating a high rate of star formation (Verstraete et al. 2001; Peeters et al. 2004; Wu et al. 2005; Calzetti et al. 2007; Bendo et al. 2008).

Another slight rise in the major-axis profile exists at much smaller radii, from $1.2 \operatorname{arcsec}^{1 / 4} \leqslant r^{1 / 4} \leqslant 1.6 \operatorname{arcsec}^{1 / 4}$ $(280 \mathrm{pc} \leqslant r \leqslant 880 \mathrm{pc})$. This feature appears to be real as three of the four data sources trace it; the 2MASS data are not useful at small radii and therefore do not cover the radius range of this shallower feature. Brightness contours of the inner $12^{\prime \prime} .9 \times 12^{\prime \prime} .9$ $(1.7 \mathrm{kpc} \times 1.7 \mathrm{kpc})$ of the NICMOS image are shown in Figure 7; the isophotes transition from elliptical to disky over the radius range indicated by the bump in the major-axis light 
Table 2

Sérsic Indices and Pseudobulge-to-total $(P B / T)$ Ratios for NGC 4565 and NGC 5746

\begin{tabular}{|c|c|c|c|c|c|c|}
\hline \multirow[t]{2}{*}{ Designation } & \multirow{2}{*}{$\begin{array}{c}v_{c}^{\mathrm{a}} \\
\left(\mathrm{km} \mathrm{s}^{-1}\right)\end{array}$} & \multicolumn{2}{|c|}{ Box Sérsic $n$} & \multicolumn{2}{|c|}{ Pseudobulge Sérsic $n$} & \multirow[t]{2}{*}{$P B / T$} \\
\hline & & Minor & Major & Minor & Major & \\
\hline NGC 4565 & $255 \pm 10^{\mathrm{b}}$ & 1 & $\ldots$ & $1.33 \pm 0.12$ & $1.55 \pm 0.07$ & $0.061 \pm 0.010$ \\
\hline NGC 5746 & $318.5 \pm 9.8^{c}$ & $1.16 \pm 0.18$ & $1.78 \pm 0.25$ & $0.99 \pm 0.08$ & $1.17 \pm 0.24$ & $0.136 \pm 0.019$ \\
\hline
\end{tabular}

Notes.

a Maximum circular velocity, corrected for the inclination angle.

b Rupen (1991).

c Kuijken \& Merrifield (1995)

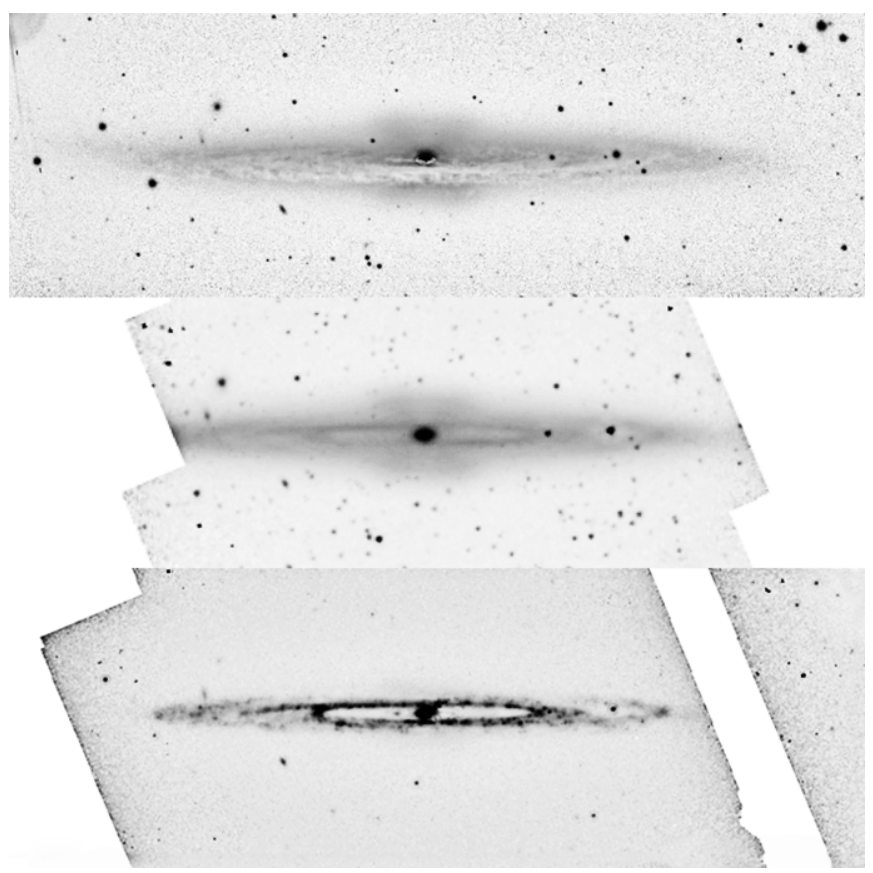

Figure 6. Three views of NGC 5746 from the optical to the mid-infrared: the sum of SDSS gri (top), Spitzer/IRAC $3.6 \mu \mathrm{m}$ (middle), and IRAC $8 \mu \mathrm{m}$ (bottom). The images have been rotated such that the major axis is aligned with the image rows. Irregularities in the IRAC images are caused by boundaries of the mosaicked regions used to make the composite image in each case.

profile. This may indicate the presence of a nuclear disk partially obscured by dust. In the region defined by elliptical isophotes, the profile is nearly linear in $r^{1 / 4}$ until it turns over at the limit of the NICMOS resolution. It is well fitted by a Sérsic function with an index very similar to that observed along the major axis of NGC 4565. We believe this is additional evidence indicating the presence of a central pseudobulge.

The major-axis light profile is consistently brighter in the SDSS optical colors between the inner bright feature and the ring over an approximate radius range of $1.8 \operatorname{arcsec}^{1 / 4} \leqslant$ $r^{1 / 4} \leqslant 2.7 \operatorname{arcsec}^{1 / 4}(1.4 \mathrm{kpc} \lesssim r \lesssim 7.1 \mathrm{kpc})$. The bluer light again dominates from $3.2 \operatorname{arcsec}^{1 / 4} \leqslant r^{1 / 4} \leqslant 3.4 \operatorname{arcsec}^{1 / 4}$ (14.0 kpc $\lesssim r \lesssim 17.9 \mathrm{kpc})$. This is roughly the radius range between the outer edge of the ring and what in the $8 \mu \mathrm{m}$ IRAC image appears to be the inner edge of a set of spiral arms. In both cases, interior to the ring and between the ring and spiral arms, the apparent blue color excess in the light profile results from starlight evenly distributed in the plane of the disk. PAH emission in the inner ring contributes as much flux in the nearand mid-IR as the starlight does, whereas in the case of the spiral arms, there are insufficient IR data at large radii to determine the relative contribution of stars and PAH emission. The last IRAC

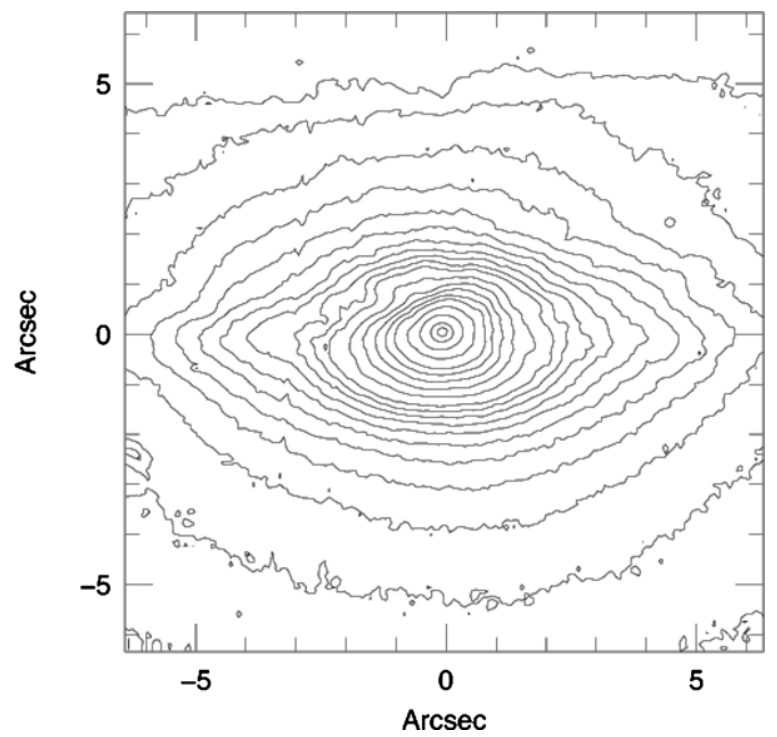

Figure 7. Brightness contour plot of the nuclear region of the NICMOS $H$-band image of NGC 5746. The image has been derotated such that the galaxy's major axis lies along the rows of the diagram and was cleaned of contaminants before the contours were generated. The contours are at pixel levels of $0.1,0.5,1,1.5,2$, $2.5,3,3.5,4,4.5,5,6,7,8,9,10,12,15,20$, and $25 \mathrm{ADU}$ after sky subtraction. The vertical and horizontal scales are arranged such that the coordinate origin corresponds to the highest pixel value in the frame. Deformation of the isophotes in the upper left quadrant is due to absorption by the dust lane.

data point in Figure 4 suggests that the profile is rising in the mid-IR as it crosses the spiral arms and would again dominate in this region if data existed. Seeing relatively unextincted starlight in the region between the inner ring and the bar is consistent with removal of gas and dust from this region, and should be observable in other inner-ring galaxies.

\section{SUMMARY}

Using archival data from multiple ground-based surveys and spacecraft missions, we carried out surface photometry on the almost-edge-on spiral galaxy NGC 5746 and extracted 1D light profiles along the galaxy's minor and major axes. The profiles were decomposed into multiple Sérsic and exponential functions corresponding to the central pseudobulge, the boxy bar, and the disk. We computed Sérsic indices for the pseudobulge, finding $n=0.99 \pm 0.08$ and $1.17 \pm 0.24$ for the minor and major axes, respectively, and an axial ratio of $\sim 0.85$. The degree of flattening of this pseudobulge is consistent with the amount we found previously for the pseudobulge in NGC 4565 . We further note that the central pseudobulge is the most compact component of the light profiles, with a scale height of only $100 \pm$ 20 pc. Near- and mid-IR imagery reveals the presence of an 
inner ring of inner radius $9.1 \mathrm{kpc}$ and width of $1.6 \mathrm{kpc}$, another structure whose presence is consistent with secular evolution. The ring and a set of outer spiral arms are clearly indicated in the major axis light profile as well. All of this implies that NGC 5746 is a well-developed, nearly edge-on SB(r)bc galaxy.

Thus, NGC 5746, like NGC 4565, is a giant galaxy whose structure shows no recognizable remnant of a recent major merger. Such galaxies are common in field environments. It remains difficult to understand how they form in a hierarchically clustering universe.

These results are based on observations made with the Spitzer Space Telescope, which is operated by the Jet Propulsion Laboratory, California Institute of Technology under a contract with NASA. Additional data from the NASA/ESA Hubble Space Telescope were used, obtained from the data archive at the Space Telescope Institute, operated by the association of Universities for Research in Astronomy, Inc. under the NASA contract NAS 5-26555. This publication makes use of data products from the Two Micron All Sky Survey, which is a joint project of the University of Massachusetts and the Infrared Processing and Analysis Center/California Institute of Technology, funded by the National Aeronautics and Space Administration and the National Science Foundation. Funding for the SDSS and SDSS-II has been provided by the Alfred P. Sloan Foundation, the Participating Institutions, the National Science Foundation, the U.S. Department of Energy, the National Aeronautics and Space Administration, the Japanese Monbukagakusho, the Max Planck Society, and the Higher Education Funding Council for England. The SDSS Web site is http://www.sdss.org/. The SDSS is managed by the Astrophysical Research Consortium for the Participating Institutions. The Participating Institutions are the American Museum of Natural History, Astrophysical Institute Potsdam, University of Basel, University of Cambridge, Case Western Reserve University, University of Chicago, Drexel University, Fermilab, the Institute for Advanced Study, the Japan Participation Group, Johns Hopkins University, the Joint Institute for Nuclear Astrophysics, the Kavli Institute for Particle Astrophysics and Cosmology, the Korean Scientist Group, the Chinese Academy of Sciences (LAMOST), Los Alamos National Laboratory, the Max-Planck-Institute for Astronomy (MPIA), the Max-Planck-Institute for Astrophysics (MPA), New Mexico State University, Ohio State University, University of Pittsburgh, University of Portsmouth, Princeton University, the United States Naval Observatory, and the University of Washington.

This work was supported by the National Science Foundation under grant AST-0607490 and by the Curtis T. Vaughan, Jr. Centennial Chair in Astronomy at the University of Texas at Austin.

Facilities: FLWO: 2MASS, Spitzer (IRAC), HST(NICMOS), Sloan

\section{REFERENCES}

Abadi, M. G., Navarro, J. F., Steinmetz, M., \& Eke, V. R. 2003, ApJ, 591, 499 Allamandola, L. J., Tielens, A. G. G. M., \& Barker, J. R. 1985, ApJ, 290, L25 Allamandola, L. J., Tielens, A. G. G. M., \& Barker, J. R. 1989, ApJS, 71, 733 Athanassoula, E. 2005, MNRAS, 358, 1477
Athanassoula, E., \& Misiriotis, A. 2002, MNRAS, 330, 35

Balcells, M., Graham, A. W., \& Peletier, R. F. 2007, ApJ, 665, 1084

Bendo, G. J., Draine, B. T., Engelbracht, C. W., et al. 2008, MNRAS, 389, 629

Bureau, M., \& Freeman, K. C. 1999, AJ, 118, 126

Bushouse, H. 1997, in The 1997 HST Calibration Workshop with a New Generation of Instruments, ed. S. Casertano, R. Jedrzejewski, T. Keyes, \& M. Stevens (Baltimore, MD: STScI), 223

Calzetti, D., Kennicutt, R. C., Engelbracht, C. W., et al. 2007, ApJ, 666, 870

Cohen, M., Wheaton, W. A., \& Megeath, S. T. 2003, AJ, 126, 1090

Combes, F., Debbasch, F., Friedli, D., \& Pfenniger, D. 1990, A\&A, 233, 82

Combes, F., \& Sanders, R. H. 1981, A\&A, 96, 164

de Vaucouleurs, G., de Vaucouleurs, A., Corwin, J., et al. 1991, Third Reference Catalogue of Bright Galaxies. Volume I: Explanations and references. Volume II: Data for galaxies between $0^{h}$ and $12^{h}$. Volume III: Data for galaxies between $12^{h}$ and $24^{h}$ (Berlin: Springer)

Fazio, G. G., Hora, J. L., Allen, L. E., et al. 2004, ApJS, 154, 10

Fisher, D. B., \& Drory, N. 2008, AJ, 136, 773

Fisher, D. B., \& Drory, N. 2011, ApJ, 733, L47

Governato, F., Brook, C., Mayer, L., et al. 2010, Nature, 463, 203

Kormendy, J. 1993, in IAU Symp. 153, Galactic Bulges, ed. H. Dejonghe \& H. J. Habing (Cambridge: Cambridge Univ. Press), 209

Kormendy, J., \& Barentine, J. C. 2010, ApJ, 715, L176

Kormendy, J., \& Bender, R. 2012, ApJS, 198, 2

Kormendy, J., Drory, N., Bender, R., \& Cornell, M. E. 2010, ApJ, 723, 54

Kormendy, J., \& Kennicutt, R. C., Jr. 2004, ARA\&A, 42, 603

Kuijken, K., \& Merrifield, M. R. 1995, ApJ, 443, L13

Léger, A., \& Puget, J. L. 1984, A\&A, 137, L5

Lupton, R., Gunn, J. E., Ivezić, Z., Knapp, G. R., \& Kent, S. 2001, in ASP Conf Ser. 238, Astronomical Data Analysis Software and Systems X, ed. F. R. Harnden, Jr., F. A. Primini, \& H. E. Payne (San Francisco, CA: ASP), 269

Merrifield, M. R. 1996, in ASP Conf. Ser. 91, IAU Colloq. 157: Barred Galaxies, ed. R. Buta, D. A. Crocker, \& B. G. Elmegreen (San Francisco, CA: ASP), 179

Merrifield, M. R., \& Kuijken, K. 1999, A\&A, 345, L47

Peebles, P. J. E., \& Nusser, A. 2010, Nature, 465, 565

Peeters, E., Spoon, H. W. W., \& Tielens, A. G. G. M. 2004, ApJ, 613, 986

Pfenniger, D., \& Friedli, D. 1991, A\&A, 252, 75

Pfenniger, D., \& Norman, C. 1990, ApJ, 363, 391

Puget, J. L., \& Léger, A. 1989, ARA\&A, 27, 161

Raha, N., Sellwood, J. A., James, R. A., \& Kahn, F. D. 1991, Nature, 352, 411

Rand, R. J., \& Benjamin, R. A. 2008, ApJ, 676, 991

Rupen, M. P. 1991, AJ, 102, 48

Ryan, R. E., Jr., Cohen, S. H., Windhorst, R. A., \& Silk, J. 2008, ApJ, 678, 751

Schweizer, F. 1990, in Interactions in Our Time, ed. R. Wielen (Berlin: Springer), 60

Sérsic, J. L. 1968, Atlas de Galaxias Australes (Cordoba: Observatorio Astronomico)

Shen, J., Rich, R. M., Kormendy, J., et al. 2010, ApJ, 720, L72

Simien, F., \& de Vaucouleurs, G. 1986, ApJ, 302, 564

Skrutskie, M. F., Cutri, R. M., Stiening, R., et al. 2006, AJ, 131, 1163

Springob, C. M., Masters, K. L., Haynes, M. P., Giovanelli, R., \& Marinoni, C. 2009, ApJS, 182, 474

Thompson, R. I. 1994, Proc. SPIE, 2209, 319

Tielens, A. G. G. M., Hony, S., van Kerckhoven, C., \& Peeters, E. 1999, in The Universe as Seen by ISO, ed. P. Cox \& M. Kessler (ESA Special Publication, Vol. 427; Noordwijk: ESA), 579

Tielens, A. G. G. M., van Kerckhoven, C., Peeters, E., \& Hony, S. 2000, in IAU Symp. 197, From Molecular Clouds to Planetary, ed. Y. C. Minh \& E. F. van Dishoeck (Cambridge: Cambridge Univ. Press), 349

Toomre, A. 1977, in Evolution of Galaxies and Stellar Populations, ed. B. M. Tinsley \& R. B. G. Larson (New Haven, CT: Yale Univ. Obs.), 401

Tully, R. B. 1988, Nearby Galaxies Catalog (Cambridge: Cambridge Univ. Press)

Tully, R. B., Rizzi, L., Shaya, E. J., et al. 2009, AJ, 138, 323

Tully, R. B., Shaya, E. J., Karachentsev, I. D., et al. 2008, ApJ, 676, 184

van der Kruit, P. C., \& Searle, L. 1981, A\&A, 95, 105

Verstraete, L., Pech, C., Moutou, C., et al. 2001, A\&A, 372, 981

White, S. D. M., \& Rees, M. J. 1978, MNRAS, 183, 341

Willick, J. A., Courteau, S., Faber, S. M., et al. 1997, ApJS, 109, 333

Wu, H., Cao, C., Hao, C.-N., et al. 2005, ApJ, 632, L79

York, D. G., Adelman, J., Anderson, J. E., Jr., et al. 2000, AJ, 120, 1579 